\title{
Peer Reviewers for the Journal of the American Board of Family Medicine in 2020
}

\author{
Marjorie A. Bowman, MD, MPA, Dean A. Seebusen, MD, MPH, \\ Christy J. W. Ledford, PhD, and Phil Lupo, MLIS
}

\section{( J Am Board Fam Med 2021;34:252-256.)}

To our peer reviewers: We thank you for your time, expertise, and dedication to the fournal of the American Board of Family Medicine ( $7 A B F M)$.

$7 A B F M$ peer reviewers serve a vital role in the field of family medicine and its scientific community. We acknowledge and thank our peer reviewers for contributing their time and expertise throughout this tumultuous year. In 2020, 617 individuals performed 696 peer reviews, regarding the suitability of articles for publication in the $7 A B F M$. It was a record-setting year for the FABFM with 651 manuscript submissions, which made each reviewer's contribution all the more valuable.

Conflict of interest: The authors are editors of the $7 A B F M$.

\section{Top Reviewers}

Each manuscript submitted to the $7 A B F M$ is first reviewed by the editors and, when deemed suitable for peer review, is evaluated and scored by volunteer reviewers. In the list below, peer reviewers with an asterisk next to their name are our top reviewers for 2020 based on the quantity of critiques provided.

\section{Seeking New Reviewers}

We also enjoy welcoming new peer reviewers into the fold. Peer review is a vital service that helps authors and researchers improve their work. Reviewers are always free to decline a request or to indicate periods of unavailability. If you would like to volunteer to review for the $7 A B F M$, please download and complete the Peer Reviewer Form, which can be accessed online at https://www.jabfm.org/content/ information-reviewers. Then, e-mail it, along with your curriculum vitae, to plupo@theabfm.org.

$\begin{array}{lll}\text { 2020 Peer Reviewers } & & \\ \text { Rohit Abraham* } & \text { Barbara S. Apgar } & \text { George R. Bergus } \\ \text { Louise S. Acheson } & \text { Thomas A. Arcury } & \text { Maresi Berry-Stoelzle } \\ \text { Sonal Admane } & \text { Christine Arenson* } & \text { Brittany A. Bettendorf* } \\ \text { Krishna A. Agarwal } & \text { Vikram Arora } & \text { Ravi Bhargava } \\ \text { David C. Agerter } & \text { Angele Arthur } & \text { Charles J. Billington } \\ \text { Alicia L. Agnoli* } & \text { Gary N. Asher } & \text { Juliann Binienda* } \\ \text { Morhaf Al Achkar } & \text { Latifi A. Asrar } & \text { Rajshekhar Bipeta } \\ \text { Sommer Aldulaimi } & \text { Elisabeth L. Backer } & \text { Richard Birtwhistle } \\ \text { Grace A. Alfonsi } & \text { Steffani R. Bailey } & \text { Alan Blum } \\ \text { Mohammad M. Alkot } & \text { Matthew G. Barnes } & \text { Debra Boardley* } \\ \text { Nishita Shah Amin } & \text { Tyler Barreto } & \text { George W. Bock* } \\ \text { Antony D. Anderson } & \text { Kirsten Barrett } & \text { John M. Boltri* } \\ \text { Kurt B. Angstman } & \text { Maren Batalden } & \text { Curtis Bone } \\ \text { Ann Gene Anthony* } & \text { Laurence Bauer } & \text { Jason C. Booza } \\ \text { Kimberly Aparicio } & \text { Ian M. Bennett* } & \text { Amanda E. Borsky }\end{array}$




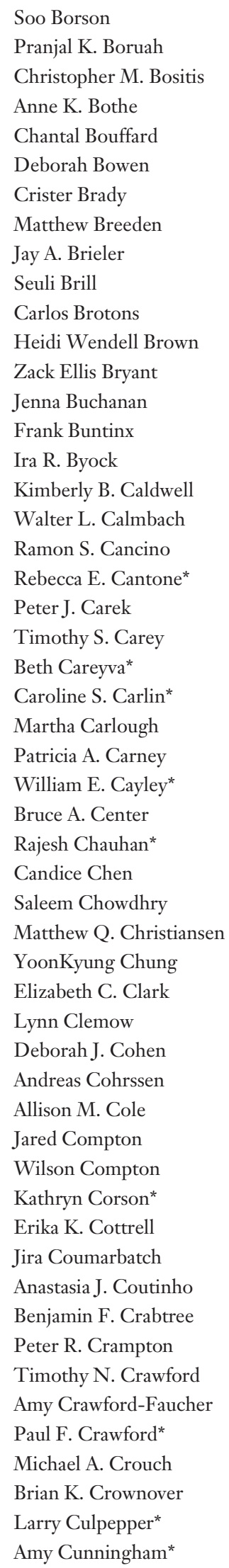

\begin{tabular}{|c|c|}
\hline Joshua Cusick-Lewis & Jonathan Foley* \\
\hline Timothy P. Daaleman* & Kanti R. Ford \\
\hline Simone Dahrouge & Gary Franklin* \\
\hline Ariella Perry Dale & Adam Franks \\
\hline Jeanette M. Daly & Daniel Jason Frasca* \\
\hline Blair G. Darney & Joshua Freeman \\
\hline Jaividhya Dasarathy & Brandi K. Freeman* \\
\hline Alan K. David & John J. Frey* \\
\hline Toren Davis Jr & Julie M. Fritz \\
\hline John W. Davis* & Yuriko Fukuta \\
\hline Philip Day & Kimberly G. Fulda \\
\hline Claudia Der-Martirosian & Kylee A. Funk \\
\hline Nishanth Dev & Ellen Funkhouser \\
\hline Vanessa Diaz & Heidi L. Gaddey \\
\hline L. Miriam Dickinson & Anne H. Gaglioti \\
\hline Katrina E. Donahue & David S. Garcia* \\
\hline Andrea Dotson & Gregory M. Garrison \\
\hline Chyke A. Doubeni* & Joseph Garry \\
\hline David J. Doukas & Brian Garvey* \\
\hline Tony Dowell & Breanna Gawrys \\
\hline Deborah Dreyfus & Eric Gayle \\
\hline Joanna Drowos & Sarah Gebauer \\
\hline Michael F. Drusano & Cynthia M. Geppert \\
\hline Maurice L. Duggins & Wray Gerard \\
\hline Dora M. Dumont & Jenenne Geske* \\
\hline Scott Eathorne & Abhijeet Ghatol \\
\hline Mark H. Ebell & Valerie J. Gilchrist \\
\hline Aimee R. Eden & Julie Gleason-Comstock \\
\hline Milton Eder & Emily M. Godfrey \\
\hline Ellen M. Einterz & Rachel Gold \\
\hline Nancy C. Elder* & Stephanie B. Gold \\
\hline John W. Ely & Debora Goetz Goldberg \\
\hline John W. Epling* & Karen M. Goldstein \\
\hline Ted Epperly & Rise B. Goldstein \\
\hline Deborah R. Erlich & Francisco J. Gomez-Clavelina \\
\hline J. I. Escobar & James Goodwin \\
\hline Anna Espinoza & Rachel J. Gorwitz \\
\hline Kelly M. Everard & Thomas Granatir \\
\hline Heather B. Fagan & David T. Grande \\
\hline Krista Farey & Lucinda Grande \\
\hline T. Michael Farley & Jocelyn R. Gravlee \\
\hline Daniel Federman & Michael E. Green \\
\hline Greg Feero & Beverly Green* \\
\hline Joshua J. Fenton* & Larry A. Green* \\
\hline Jeanne $M$. Ferrante* & Grant M. Greenberg \\
\hline Scott A. Fields & Kim Griswold \\
\hline Edgar Figueroa & Michael Grover \\
\hline Kenneth S. Fink* & Alexander B. Guirguis \\
\hline Kevin Fiscella & Karen Gunning \\
\hline Bruce D. Fisher & David L. Hahn* \\
\hline Judith A. Fisher & Scott Haldeman \\
\hline Margaret M. Flinter* & Jacqueline R. Halladay \\
\hline Kory Floyd* & Robert M. Hamm \\
\hline
\end{tabular}


E. Haneke

Lee Hargraves

Kathryn Harmes

Diane M. Harper

Susan Hart-Hester

Kelly Haskard Zolnierek

Brigit Hatch

Elham Hatef

Diana L. Heiman

Margaret Helton

Jennifer Hemler

John A. Hermos

Christopher Heron

Paul J. Hershberger

Rachel Hess

Vincent W. Hevern*

Daniel Hinthorn

Jakub P. Hlavka

Courtney Hobza*

Neil R. Holland

Christina T. Holt

James D. Holt

Christian Hopfer

Michael A. Horst

Susan Hsieh

Clarissa Hsu

Christine Huang

Brenda L. Hudson

William J. Hueston

Lauren S. Hughes

Nathalie Huguet

Stephen Hulkower

Laura Hurley

Katherine Hurst

Jihad Irani

Jamal Islam

Henry R. Ivey

Yalda Jabbarpour

James Janisse

Muhammad A. Javed*

Nan Jiang

Masahito Jimbo

Michael Johansen

Jean E. Johnson

Pamela Jo Johnson

Mark S. Johnson*

Christopher E. Jonas*

Roger Jones

Linda S. Kahn*

Neelima Kale

Sukhjeet Kaur Kamboj

Medha Karmarkar

David J. Katzelnick
Ryan Kauffman

Neil S. Kaye

Lewis Kazis

Michelle K. Keating

Robert D. Keeley

Kelly J. Kelleher

Claire Kendall

Patrick L. Kerr

Rodger Kessler*

Laila Khalid

Omar A. Khan

Birgit Khandalavala

Niharika Khanna

Morteza Khodaee

Autumn M. Kieber-Emmons

Kim S. Kimminau

Anne King

Dana E. King

Stella King

Michelle Klawans

Kyle Knierim

Tadashi Kobayashi*

Richie Kohli

Elin C. Kondrad

David E. Koren

Kelly G. Koren

Neil Korsen

Richard L. Kravitz

Alex H. Krist

John Krueger

Irina Kudrina

Jennifer Kusch

Anton J. Kuzel

Bethany M. Kwan

Michelle Lampman

Jason S. Lanham

Paul Drake Lavender

Ross A. Lawrenson

Michael LeFevre

Patricia M. Lenahan

Robert P. Lennon

Joanne Leovy*

Lenard I. Lesser*

Cari Levy

Douglas Levy

Sonja M. Likumahuwa-Ackman

Susan X. Lin

Deerajnath Lingutla

Mark Linzer

Christos Lionis

Benjamin Littenberg*

Sahoko H. Little

Michael A. Liu
Mark Loafman

Natalia Loskutova

Jody Lounsbery

Jorgen Lous

David A. Loxterkamp*

Sean C. Lucan*

Hillary D. Lum*

Diane J. Madlon-Kay

Elizabeth Magnan*

Rohan Mahabaleshwarkar*

Megan R. Mahoney

Martin C. Mahoney*

Arch G. Mainous

Mark Makowsky

Rebecca A. Malouin

David M. Mannino

Katherine Margo

David Margolius

Jason E. Marker

Amanda Markovitz

John Marley

Emily G. Marshall

Cara Marshall*

Patricia Martin

James Massey

Eric Mast

Samuel C. Matheny

Maureen Mayhew

Ann S. McAlearney

Una D. McCann

Catherine McCarthy

Adelaide McClintock

Susan H. McDaniel

Patrick S. McFarlane

Brian McKinstry

David R. Mehr

Taverna Melanie

Oxana M. Mian*

Aaron Michelfelder

Lloyd Michener*

Donald B. Middleton

William Miller*

Logan Mims

Tasaduq Mir

Ranit Mishori

Kei Miyazaki

Jane Moeckli

Tania Moerenhout

Kimberly Mohabir

Jennifer Molokwu

Miranda Moore*

Roy N. Morcos*

Laura Morgan-Frankart 
Sherri Lynn Morgan*

Christopher P. Morley

Cortney M. Mospan

Karen Muchowski

John P. Muench

Elie Mulhem

Pamela A. Mund

Rahma Mungia

William J. Murdoch

Joseph M. Murley

Timothy Myrick

Zsolt J. Nagykaldi*

Mohammad Obadah Nakawah

Priya Nambisan

Laeth S. Nasir

Terence Dwight Naumann

Victoria Neale

Donald E. Nease

Andrea Lynn Nederveld

Lynne S. Nemeth

Warren P. Newton*

Len Nichols

Kari Nilsen

Thomas E. Norris*

Mary Patricia Nowalk

Cormac John O'Connor*

Catherine A. O'Donnell

Jason O'Grady

David T. O'Gurek

Thomas R. O'Neill

Ebiere Okah

J. Nwando Olayiwola

Mariusz Olszewski

Emily Onello

Eriko Onishi

Sarah S. Ono

Amimi Sandra Osayande

Angulique Y. Outlaw

Darrell R. Over

Wilson D. Pace

Janet Page

Jina Pakpoor

Heather Paladine

Scott A. Paluska

John M. Pascoe

Darshan J. Patel

Donald Pathman*

Nikita Patil

Jyothi R. Patri

Colleen Payton

Kevin A. Pearce

Randolph L. Pearson

Brian R. Penti

Allen Perkins

Leigh Perreault
Kevin A. Peterson

Lars Peterson

Stephen Petrany

Darcy F. Phelan-Emrick

William R. Phillips*

Stephen Pickett

Andrew D. Pinto

Lakshmi Polavarapu

Andrew Pomerantz

Charles D. Ponte*

Thejaswi Poonacha

M. C. Post

Michael B. Potter*

Stacy E. Potts

P. Prasadarao

David W. Price*

Elizabeth A. Quigley

Howard K. Rabinowitz

Ravishankar Ramaswamy

Stephen Ratcliffe

Brian V. Reamy

Nicole Reichhart

Chelsea E. Renfro

Peter L. Reynolds

Robert L. Rhyne

Thomas C. Ricketts

Jill Rissi

Peter A. Rives*

William Roberts

Sean Robinson

Pamela G. Rockwell

Michelle A. Roett

Michael Roizen

Adam J. Rose

Danielle E. Rose

Thomas C. Rosenthal

Robert G. Ross

Walter Rosser

Muni Rubens

Mack T. Ruffin*

Terry S. Ruhl

David Rushlow

Tracy A. Rydel

Roy T. Sabo

Saria C. Saccocio

Carolyn J. Sachs*

Joseph Sakai

Ashkan Salamatipour

Enrique Sanchez Valdivieso

Paulo A. Santos

Amit Sapra

David Satin

Amelia Sattler

Judith A. Savageau

Ghanshyambhai T. Savani
Barry G. Saver

Margot Savoy

Ramesh Saxena

Robert M. Schainfeld

Joseph E. Scherger

Carolina Gonzalez Schlenker

F. David Schneider

Sarina Schrager*

Kendra Schwartz*

Miriam E. Schwartz*

Thomas L. Schwenk

Kevin Selby

Jessica Servey*

Richard K. Severson

Patricia S. Sexton

Patricia L. Seymour

Anjana E. Sharma

Manisha Sharma

Eric K. Shaw

Jonathan Shaw

Kayce Shealy

Scott A. Shipman

Saima Siddiqui

Cynthia J. Sieck

Victor S. Sierpina

Donald Silberberg

Allison Silveus

Gina Simoncini

Matthew Simpson

Vijay Singh

Paul A. Sloan

David S. Smith

Jeanene Smith

Leif I. Solberg

Todd D. Sorensen

Ivan Sosa

Tulay G. Soylu*

John G. Spangler

Sukanya Srinivasan

Yazhini Srivathsal

Joshua St. Louis

Michael Stehney

Elizabeth Steiner

Kari A. Stephens

Mark B. Stephens

Earl Ray Stewart

Melissa Stiles

Mark L. Stovak

Scott M. Strayer

George D. Strelioff

Courtney Kimi Suh

Jennifer L. Sullivan

Walton Sumner

Bei Sun

Jeff L. Susman 
Kamala Swayampakala

David E. Swee

Daniel J. Tancredi

Hazel Tapp*

Derjung M. Tarn

Yhenneko J. Taylor

Deanna E. Telner

Chris M. Terpening

Matthew Thompson

Manish M. Tiwari

Katherine H. Tkaczuk

Barbara Tobias

Sophia Tolliver

Rachelle Toman

Sebastian T. Tong*

Stefan Topolski

John M. Tovar

Howard Tracer

Dennis Tsilimingras

Marc Tunzi

Joanne Turnbull

Maria Ukhanova

Divvy Upadhyay

Mark J. Upfal

Carole C. Upshur

Anthony F. Valdini

George Valko

Daniel J. Van Durme

David Vearrier

Carla Veiga Rodrigues
William B. Ventres

Mohamud A. Verjee

Annapia Verri

Bonnie M. Vest

Varsha G. Vimalananda

Nicki Vithalani

Kirsten Vitrikas

Gordon Walbroehl

Eric Wall

Lorraine S. Wallace

Neal T. Wallace

Judith M. E. Walsh

Brian W. Ward

Lisa M. Ward

Richard C. Wasserman

Jason A. Waterman

J. Todd Weber

Barry D. Weiss

Toney Welborn

David L. Weldy

Mary Jo Welker

Richard C. Wender

Andrea Wendling*

Emily Wentzell

Jack M. Westfall*

Randy K. Wexler

Karen M. Whalen

Luke A. White

John Sharkey White*

Elizabeth Wickersham
Marcy Wiemers

Elizabeth A. Wiley

Rachel Willard-Grace*

Summer Williams

Jonathan D. Winter

Diana Wohler

L. Thomas Wolff

Jeffrey Wolfrey

Michelle Wong

Anne B. Woods

Justine P. Wu

Imam M. Xierali*

Jinping Xu

Zhijie Xu

Mark J. Yaffe

Olga Yakusheva

Bektas Murat Yalcin

Joseph R. Yancey

Mark W. Yeazel

Alisa P. Young

Richard A. Young

Rodney B. Young

Jonathan Yun

Leanne Zakrzewski

Jessica R. Zarndt

Philip Zazove

Min Zhang

Emily B. Zimmerman

Samuel H. Zuvekas

*Top reviewers in 2020 (based on quantity and quality of peer reviews written).

To see this article online, please go to: http://jabfm.org/content/34/2/252.full. 